\title{
Paradoxical increases in serum levels of highly chlorinated $P C B s$ in aged women in clear contrast to robust decreases in dietary intakes from 1980 to 2003 in Japan
}

\author{
Akio Koizumi - Kouji H. Harada Bita Eslami Yoshinori Fujimine Noriyuki Hachiya \\ Iwao Hirosawa · Kayoko Inoue · Sumiko Inoue · Shigeki Koda • Yukinori Kusaka • Katsuyuki Murata • \\ Kazuyuki Omae · Norimitsu Saito - Shinichiro Shimbo · Katsunobu Takenaka - Tatsuya Takeshita • \\ Hidemi Todoriki $\cdot$ Yasuhiko Wada $\cdot$ Takao Watanabe $\cdot$ Masayuki Ikeda
}

Received: 27 October 2008/Accepted: 26 April 2009/Published online: 28 May 2009

(C) The Japanese Society for Hygiene 2009

\begin{abstract}
Objective Exposure to polychlorinated biphenyls (PCBs) is considered to have culminated between 1950 and 1970 in Japan, and exposure through diet, the major exposure route, has decreased significantly over the last 10 years. The primary goal of the present study was to investigate the long-term trends and congener profiles of serum and dietary levels of PCBs using historical samples.
\end{abstract}

A. Koizumi $(\bowtie) \cdot$ K. H. Harada · B. Eslami · K. Inoue

S. Inoue

Department of Health and Environmental Sciences,

Graduate School of Medicine, Kyoto University,

Yoshida Konoe, Sakyo, Kyoto 606-8501, Japan

e-mail: koizumi@pbh.med.kyoto-u.ac.jp

Y. Fujimine

Otsuka Pharmaceutical Company Ltd,

Tokushima 771-0915, Japan

N. Hachiya

National Institute for Minamata Disease,

Minamata 867-0055, Japan

I. Hirosawa

Kansai University of Welfare Sciences,

Osaka 582-0026, Japan

S. Koda

National Institute of Occupational Safety and Health,

Japan, Kawasaki 214-8585, Japan

Y. Kusaka

School of Medicine, University of Fukui,

Matsuoka 910-1193, Japan

K. Murata

Akita University School of Medicine, Akita 010-8543, Japan
Methods Using banked samples collected in 1980, 1995, and 2003 surveys, we determined the daily intakes and serum concentrations of 13 PCB congeners (\#74, \#99, \#118, \#138, \#146, \#153, \#156, \#163, \#164, \#170, \#180, \#182, and \#187) in women.

Results The total daily PCB intake [ng/day, geometric mean (geometric standard deviation)] decreased significantly from 523 (2.5) in 1980 to 63 (3.2) in 2003. The

\section{K. Omae}

School of Medicine, Keio University, Tokyo 160-8582, Japan

N. Saito

Research Institute for Environmental Sciences and Public

Health of Iwate Prefecture, Morioka 020-0852, Japan

S. Shimbo

Kyoto Women's University, Kyoto 605-8501, Japan

K. Takenaka

Takayama Red Cross Hospital, Takayama 506-8550, Japan

T. Takeshita

Wakayama Medical University, Wakayama 641-8509, Japan

H. Todoriki

School of Medicine, University of the Ryukyus,

Nishihara 903-0215, Japan

Y. Wada

Japan Labour Health and Welfare Organization,

Kansai Rosai Hospital, Amagasaki 660-8511, Japan

T. Watanabe

Miyagi University of Education, Sendai 980-0845, Japan

M. Ikeda

Kyoto Industrial Health Association, Kyoto 604-8472, Japan 
serum total PCB level (ng/g lipid) in women $<40$ years of age decreased significantly from 185 (1.8) in 1980 to 68 (1.8) in 2003. In contrast, the level in women $>50$ years of age increased significantly from 125 (1.7) in 1980 to 242 (1.7) in 2003. Specifically, the serum concentrations of hexa (\#138, \#146, \#153, \#156, \#163, and \#164) and hepta (\#170, \#180, \#182, and \#187) congeners increased significantly. A comparison of the serum PCB levels of women born from 1940 to 1953 revealed that their serum total PCB level was significantly higher in the 2003 survey [242 (1.7), $n=9$ ] than in the 1995 [128 (2.0), $n=17]$ surveys. This increase in the total PCB level was attributable to increases in the hepta congener groups.

Conclusion Present results suggest a decreased rate of elimination of hepta congeners with aging in females, rather than a birth-generation phenomenon.

Keywords Polychlorinated biphenyl - Congener profiles · Diet $\cdot$ Serum · Aging · Decrease in metabolism

\section{Introduction}

Polychlorinated biphenyls (PCBs), which were produced from 1930 to the $1970 \mathrm{~s}$, are still found in the environment. Although human exposure to PCBs has been persistent, it has recently decreased significantly $[1,2]$. Although the mechanisms of this reduction are still unknown, it is speculated to be due to degradation and/or diffusion under the control of global dynamics [3].

We recently established a human specimen bank for monitoring long-term exposure to persistent organic pollutant (POPs). The seeds of the sample bank were collected by Prof. Ikeda and his colleagues in the 1980s and 1990s $[4,5]$. Samples newly collected in 2003-2004 have now been added to expand the quantities available in the bank.

Using samples in the human specimen bank from the 1980s and 1990s, we previously showed that exposure to PCBs through the dietary route decreased significantly in the mid-1990s [6]. However, it remains unanswered whether there are long-term decreasing trends for the serum and daily intake levels of PCBs.

The human PCB body burden reflected by the serum congener profile is an integrated function of various factors, including the source, route, timing of exposure, and individual determinants of metabolism and clearance [7]. An age-associated increase in the PCB body burden has been emerging as a common phenomenon in many countries in recent studies [8-11]. However, most of the observations have been based on samples collected at a single time point. Exposure to PCBs is considered to have culminated between 1950 and 1970 in Japan. Therefore, generations born between 1940 and 1960 are considered to have been exposed to PCBs from childhood to young adulthood, while generations born after 1970 are thought to have experienced less exposure to PCBs due to the banning of PCB production and use in Japan in the 1973. However, it remains uncertain whether the observed age-associated changes in the levels and profiles of serum PCBs represent a generation phenomenon or are associated with decreased elimination or food preferences.

PCBs are known to be metabolized to their phenolic counterparts through dehalogenation and hydroxylation by P450s [12-15]. These counterparts are generally more hydrophilic than the parent compounds and are therefore more easily eliminated from the body than the parent PCB congeners [12]. The metabolic activity mediated by $\mathrm{P} 450$ is known to decrease with age [16]. Therefore, there might be a possibility that the timing of exposure, metabolism, and dietary profiles of PCB congeners are major factors that determine the serum levels of PCBs.

The primary goal of the present study was to investigate the long-term trends and congener profiles of the serum and dietary levels of PCBs using historical samples. The samples analyzed were collected in three surveys of different periods between 1980 and 2003. All three surveys contained generations that were born between 1940 and 1953 . These generations are expected to provide further insights into the long-term trend for the human body burden of PCBs.

\section{Materials and methods}

Target population, serum samples, and food samples

Serum and food samples collected from 1977 to 1981 (the 1980 survey) [5], 1991 to 1997 (the 1995 survey) [4], and 2003 to 2004 (the 2003 survey) have been stored in our sample bank. The protocol for sample collection in the 1980 and 1995 surveys has been documented previously [6]. Samples were collected from both males and females. In the 1980 and 1995 surveys, serum and food samples were collected from 1977 to 1981 and 1991 to 1997 , respectively, in eight prefectures in eight districts: Hokkaido, Miyagi (Tohoku), Gunma (Kanto), Ishikawa (Chubu), Shimane (Chugoku), Kochi (Shikoku), Kagoshima (Kyushu), and Okinawa. In these surveys, the participants donated serum as well as duplicate portions of the food that they had consumed over the previous $24 \mathrm{~h}$. In the 2003 survey, we collected serum samples and meals in eight prefectures in six districts: Akita and Miyagi (Tohoku), Fukui and Gifu (Chubu), Osaka and Kyoto (Kansai), Yamaguchi (Chugoku), Kochi (Shikoku), and Okinawa. Serum samples were donated when the subjects attended an annual health checkup. Information was 
limited to age and gender. We collected trios of meals (breakfast, lunch, and dinner) with water $(1.5 \mathrm{~L})$ each day in the spring and fall seasons. The combination of breakfast, lunch, and dinner was arranged so that the food menus followed the most common consumption patterns of subjects aged between 30 and 60 years in the local community areas in the season of sampling. A total of 50 trios and water were purchased from a local commercial vendor in each sampling site. Each trio of meals was carefully homogenized with the $1.5 \mathrm{~L}$ of water to prevent contamination and a portion of the homogenate was stored at $-20^{\circ} \mathrm{C}$ in two $1-\mathrm{L}$ polypropylene tubes.

Blood samples were taken from a cubital vein late in the morning. Serum samples were separated by centrifugation at $1,500 \times g$ for $15 \mathrm{~min}$, and stored at $-20^{\circ} \mathrm{C}$. All samples have been stored in the Department of Health and Environmental Sciences, Kyoto University Graduate School of Medicine. Quality controls for contamination monitoring were checked as previously described [6].

The study population of the present study was limited to female participants because sampling in the 2003 surveys was limited to females. We randomly selected participants from each sampling site.

A verbal form of informed consent was obtained for the 1980 and 1995 surveys and written informed consent was obtained for the 2003 survey. Participation in the surveys was completely voluntary. In 2003, we obtained approval for delivery and analysis of the serum samples from the Ethics Committee of the Kyoto University Graduate School of Medicine on 14 November 2003 (E25).

\section{Determination of PCBs}

We determined the concentrations of 13 PCB congeners (IUPAC \#74, \#99, \#118, \#138, \#146, \#153, \#156, \#163, $\# 164, \# 170, \# 180$, \#182, and \#187) in the serum and food homogenates. In the present study, we classified the tetra congener (\#74) and penta congeners (\#99 and \#118) as penta CBs, hexa congeners (\#138, \#146, \#153, \#156, \#163, and \#164) as hexa CBs, and hepta congeners (\#170, \#180, $\# 182$, and \#187) as hepta CBs. These congeners were selected because they represent the most predominant congeners in the environment $[2,17,18]$. Determination of these congeners was carried out as previously reported [19]. Briefly, $1 \mathrm{~mL}$ serum was used to determine the serum levels of the PCBs. For PCBs in food, $2 \mathrm{~g}$ food homogenate was used. For sample blanks and tube extracts, five samples were used for each preparation. High-resolution gas chromatography/high-resolution mass spectroscopy (HRGC/HRMS), which consisted of an AutoSpec-Ultima (Micromass, UK) and a HP-6890 Series gas chromatograph (Agilent Technologies, Inc., USA), was used for analysis.
The column used was a HT8-PCB capillary column (0.25 mm I.D. × 60 m; Kanto Chemical Co., Inc., Japan). The analytical conditions used were described previously [19].

All analyzes were quantified by the isotope dilution method. The recovery of spiked internal standards was calculated against the spikes. The limit of detection (LOD) and the limit of quantification (LOQ) for each PCB congener were 0.02 and $0.2 \mathrm{pg} / \mathrm{ml}$, respectively.

The serum levels of PCBs are expressed as ng/g lipid [20]. The serum total cholesterol and triglyceride levels were measured using an aliquot of each serum sample [21]. The daily intakes of PCBs in food per person are expressed as ng/day.

\section{Statistical analysis}

The concentrations of PCBs showed log-normal distributions and were log-transformed for all analyzes. The PCB concentrations are presented as geometric mean (GM) with geometric standard deviation (GSD). Where appropriate, arithmetic mean \pm standard deviation $(\mathrm{M} \pm \mathrm{SD})$, range, medians, 25th percentile, and 75th percentile are also shown.

Database management and all statistical analyzes were performed with SAS software (version 8.2; SAS Institute). Analysis of variance (ANOVA) was used for all analyzes. A $p$ value of less than 0.05 was considered to be statistically significant.

\section{Results}

Sampling time and demographic features of the participants

Variation of sampling periods is shown in Table 1. A total of 40 serum samples from each of the 1980 and 1995 surveys and 90 serum samples from the 2003 survey were analyzed. For the 1980 and 1995 surveys, the samples were randomly selected for five participants per sampling site. For the 2003 survey, ten serum samples were randomly selected for each sampling site. We failed in the preparation of one serum sample for determination, collected in Hokkaido in 1980.

The mean ages (SD) and birth years (SD) are shown in Table 1. Height, body weight, and BMI were available only for the 1980 and 1995 surveys. The mean birth years were 1937.9 (9.8), 1942.1 (9.7), and 1966.8 (9.9) in the 1980, 1995, and 2003 surveys, respectively. The sample recoveries were more than $97 \pm 2 \%(n=5)$. All five sample blanks and tube extracts were found to be less than the LOD. 
Table 1 Demographic factors of the study participants

\begin{tabular}{|c|c|c|c|c|}
\hline $\begin{array}{l}\text { Survey year } \\
\text { No. }\end{array}$ & $\begin{array}{l}1980 \\
39^{\mathrm{a}}\end{array}$ & $\begin{array}{l}1995 \\
40\end{array}$ & $\begin{array}{l}2003 \\
90\end{array}$ & $\begin{array}{l}\text { Total } \\
169\end{array}$ \\
\hline \multicolumn{5}{|c|}{ Sampling period } \\
\hline Mean \pm SD & $1980.4 \pm 0.7$ & $1995.3 \pm 0.8$ & $2003 \pm 0$ & $1987.8 \pm 7.5$ \\
\hline Range & 1979-1981 & 1994-1997 & 2003 & 1979-2003 \\
\hline Q1 & 1979 & 1994 & - & 1980 \\
\hline Q2 & 1981 & 1995 & - & 1988 \\
\hline Q3 & 1981 & 1996 & - & 1995 \\
\hline \multicolumn{5}{|l|}{ Age (years) } \\
\hline Mean \pm SD & $42.4 \pm 9.9$ & $53.2 \pm 10.1$ & $36.2 \pm 9.9$ & $41.7 \pm 12.0$ \\
\hline Range & $24-65$ & $37-76$ & $20-58$ & $20-76$ \\
\hline Q1 & 37 & 44 & 29 & 32 \\
\hline Q2 & 41 & 55 & 35 & 40 \\
\hline Q3 & 51 & 61 & 44 & 50 \\
\hline \multicolumn{5}{|l|}{ Birth year } \\
\hline Mean \pm SD & $1937.9 \pm 9.8$ & $1942.1 \pm 9.7$ & $1966.8 \pm 9.9$ & $1954.1 \pm 16.7$ \\
\hline Range & 1916-1957 & 1920-1958 & $1945-1983$ & 1916-1983 \\
\hline Q1 & 1930 & 1935 & 1959 & 1941 \\
\hline Q2 & 1940 & 1941 & 1969 & 1954 \\
\hline Q3 & 1944 & 1951 & 1974 & 1969 \\
\hline \multicolumn{5}{|l|}{ Height $(\mathrm{cm})$} \\
\hline Mean \pm SD & $150.5 \pm 6.6$ & $152.5 \pm 5.6$ & NA & NA \\
\hline Range & $136.7-164.5$ & $138.6-162.5$ & NA & NA \\
\hline Q1 & 136.7 & 138.6 & NA & NA \\
\hline Q2 & 150.0 & 152.7 & NA & NA \\
\hline Q3 & 154.9 & 157.1 & NA & NA \\
\hline \multicolumn{5}{|c|}{ Body weight (kg) } \\
\hline Mean \pm SD & $53.4 \pm 7.1$ & $57.6 \pm 9.8$ & NA & NA \\
\hline Range & $43.5-72.0$ & $42.8-78.2$ & NA & NA \\
\hline Q1 & 43.5 & 42.8 & NA & NA \\
\hline $\mathrm{Q} 2$ & 52.0 & 55.6 & NA & NA \\
\hline Q3 & 59.0 & 62.2 & NA & NA \\
\hline \multicolumn{5}{|l|}{ BMI $\left(\mathrm{kg} / \mathrm{m}^{2}\right)$} \\
\hline Mean \pm SD & $23.6 \pm 2.8$ & $24.8 \pm 4.2$ & NA & NA \\
\hline Range & $19.5-31.8$ & $18.0-33.6$ & NA & NA \\
\hline Q1 & 24.8 & 27.0 & NA & NA \\
\hline Q2 & 23.9 & 23.9 & NA & NA \\
\hline Q3 & 21.9 & 21.6 & NA & NA \\
\hline
\end{tabular}

${ }^{\text {a }}$ One sample was lost during preparation

$N A$ not available
The distribution patterns of the three isomer groups were similar among the three surveys and the ratios of hexa/ penta (about 2) and hepta/penta (about 0.8) congeners remained unchanged among the three periods.

Age and congener patterns of PCBs in serum during 1980 and 2003

The total PCB levels in the serum did not differ according to age group in either the 1980 or 1995 survey. In contrast, a significant age-associated increase became evident in the 2003 survey, since the PCB level increased from 68 to 242 
Table 2 Intake of PCBs in diet per day

\begin{tabular}{|c|c|c|c|c|c|c|c|c|c|c|}
\hline \multirow{2}{*}{$\begin{array}{l}\text { Survey year } \\
\text { No. } \\
\text { Age group (years) }\end{array}$} & \multicolumn{4}{|l|}{1980} & \multicolumn{4}{|l|}{1995} & \multirow{2}{*}{$\begin{array}{l}2003 \\
80\end{array}$} & \multirow{2}{*}{$\begin{array}{l}\text { Total } \\
160\end{array}$} \\
\hline & 40 & $\begin{array}{l}(17) \\
(<40)\end{array}$ & $\begin{array}{l}(13) \\
(40-50)\end{array}$ & $\begin{array}{l}(10) \\
(>50)\end{array}$ & 40 & $\begin{array}{l}(2)^{\mathrm{a}} \\
(<40)\end{array}$ & $\begin{array}{l}(17) \\
(40-50)\end{array}$ & $\begin{array}{l}(21) \\
(>50)\end{array}$ & & \\
\hline \multicolumn{11}{|c|}{ Total PCBs (ng/g lipid) } \\
\hline GM (GSD) & $523(2.5)^{*}$ & $467(2.7)$ & $703(2.3)$ & $431(2.4)$ & $166(3.3)^{*}$ & $138(13.3)$ & $215(2.4)$ & $137(3.8)$ & $63(3.2)^{*}$ & $137(4.1)$ \\
\hline Range & $105-3412$ & $112-3412$ & $227-3206$ & $105-1707$ & $5.7-1548$ & $22-863$ & $32-1548$ & $5.7-1026$ & $5.5-1102$ & $5.5-3412$ \\
\hline Q1 & 283 & 219 & 344 & 255 & 85 & - & 158 & 55 & 30 & 41 \\
\hline Q2 & 510 & 503 & 603 & 468 & 196 & - & 215 & 154 & 52 & 139 \\
\hline Q3 & 925 & 865 & 1205 & 737 & 408 & - & 368 & 432 & 129 & 450 \\
\hline \multicolumn{11}{|l|}{ CB-74 (ng/g lipid) } \\
\hline GM (GSD) & $19(2.6)^{*}$ & $16(2.6)$ & $29(2.4)$ & $14(2.4)$ & $6.4(2.6)^{*}$ & $6.3(7.7)$ & $8.0(1.8)$ & $5.9(3.1)$ & $2.6(3.1)^{*}$ & $5.4(3.8)$ \\
\hline Range & $3.1-165$ & $3.1-79$ & $9.4-165$ & $4.2-54$ & $1.0-43$ & $1.5-27$ & $2.4-19$ & $1.1-43$ & $0.1-38$ & $0.1-165$ \\
\hline Q1 & 10.1 & 8.1 & 13 & 5.7 & 3.5 & - & 5.0 & 2.5 & 1.4 & 2.2 \\
\hline Q2 & 20 & 16 & 28 & 17 & 7.3 & - & 7.6 & 6.6 & 2.6 & 5.0 \\
\hline Q3 & 32 & 32 & 40 & 31 & 13 & - & 13 & 14 & 4.7 & 14 \\
\hline \multicolumn{11}{|l|}{ CB-99 (ng/g lipid) } \\
\hline GM (GSD) & $35(2.5)^{*}$ & $30(2.5)$ & $53(2.3)$ & $28(2.5)$ & $15(2.5)^{*}$ & - & $16(2.0)$ & $13(2.8)$ & $5.6(3.4)^{*}$ & $11(3.8)$ \\
\hline Range & $7.0-263$ & $7.0-134$ & $18-263$ & $8.3-118$ & ND-66 & ND-66 & $3.3-59$ & ND-61 & ND-116 & ND-263 \\
\hline Q1 & 19 & 13 & 23 & 11 & 6.5 & - & 10.5 & 4.1 & 2.8 & 4.2 \\
\hline Q2 & 39 & 36 & 53 & 34 & 17 & - & 18 & 15 & 5.2 & 11 \\
\hline Q3 & 68 & 59 & 84 & 52 & 26 & - & 26 & 26 & 10.8 & 29 \\
\hline \multicolumn{11}{|l|}{ CB-118 (ng/g lipid) } \\
\hline GM (GSD) & $66(2.7)^{*}$ & $55(2.9)$ & $96(2.3)$ & $56(2.6)$ & $21(3.4)^{*}$ & $19(16.6)$ & $29(2.3)$ & $16(3.7)$ & $8.3(3.4)^{*}$ & $18(4.2)$ \\
\hline Range & $8.4-438$ & $8.4-354$ & $37-438$ & $123-256$ & $0.7-139$ & $2.6-139$ & $3.5-139$ & $0.7-114$ & $0.5-160$ & $0.5-438$ \\
\hline Q1 & 37 & 22 & 42 & 28 & 9.7 & - & 20 & 5.2 & 4.2 & 5.6 \\
\hline Q2 & 74 & 76 & 94 & 63 & 29 & - & 32 & 20 & 7.0 & 16 \\
\hline Q3 & 125 & 106 & 164 & 106 & 47 & - & 47 & 47 & 16 & 57 \\
\hline \multicolumn{11}{|l|}{ CB-138 (ng/g lipid) } \\
\hline GM (GSD) & $78(2.5)^{*}$ & $68(2.7)$ & $106(2.3)$ & $65(2.4)$ & $25(3.5)^{*}$ & $23(12.8)$ & $34(2.5)$ & $19(4.1)$ & $9.4(3.2)^{*}$ & $20(4.2)$ \\
\hline Range & $13-461$ & $13-461$ & $34-435$ & $15-273$ & $0.6-255$ & $3.7-137$ & $4.9-255$ & $0.6-175$ & $1.1-143$ & $0.6-461$ \\
\hline Q1 & 44 & 32 & 53 & 35 & 12 & - & 24 & 9 & 4.2 & 5.9 \\
\hline Q2 & 80 & 82 & 92 & 69 & 30 & - & 33 & 23 & 7.3 & 21 \\
\hline Q3 & 152 & 128 & 201 & 111 & 69 & - & 57 & 67 & 20 & 69 \\
\hline \multicolumn{11}{|l|}{ CB-146 (ng/g lipid) } \\
\hline GM (GSD) & $20(2.6)^{*}$ & $17(2.8)$ & $28(2.3)$ & $17(2.6)$ & $6.4(3.9)^{*}$ & $5(20.1)$ & $8(3.1)$ & $4.9(4.2)$ & $2.2(4.4)^{*}$ & $5.0(5.0)$ \\
\hline Range & $2.9-147$ & $4.0-137$ & $9.0-147$ & $2.9-65$ & $0.4-81$ & $0.6-41$ & $0.8-81$ & $0.4-55$ & ND-61 & ND-147 \\
\hline Q1 & 9.8 & 8.1 & 14 & 9.3 & 2.9 & - & 5.6 & 2.0 & 1.0 & 1.5 \\
\hline Q2 & 21 & 15 & 27 & 21 & 8.1 & - & 8.3 & 5.7 & 1.7 & 5.2 \\
\hline Q3 & 35 & 32 & 46 & 30 & 18 & - & 18 & 18 & 5.0 & 19 \\
\hline \multicolumn{11}{|l|}{ CB-153 (ng/g lipid) } \\
\hline GM (GSD) & $135(2.6)^{*}$ & $121(2.8)$ & $182(2.3)$ & $110(2.4)$ & $44(3.5)^{*}$ & $36(13.9)$ & $57(2.6)$ & 37 (3.9) & $16(3.4)^{*}$ & $35(4.3)$ \\
\hline Range & $23-944$ & 23-944 & $58-836$ & $26-443$ & $2.0-468$ & $5.7-235$ & $7.6-468$ & $2.0-296$ & $0.6-297$ & $0.6-944$ \\
\hline Q1 & 73 & 58 & 89 & 66 & 20 & - & 42 & 16 & 6.8 & 10.1 \\
\hline Q2 & 127 & 124 & 154 & 120 & 51 & - & 56 & 43 & 13 & 35 \\
\hline Q3 & 244 & 238 & 330 & 189 & 114 & - & 106 & 113 & 33 & 115 \\
\hline \multicolumn{11}{|l|}{ CB-156 (ng/g lipid) } \\
\hline GM (GSD) & $10(2.4)^{*}$ & $9(2.6)$ & $13(2.4)$ & $8(2.3)$ & $3.9(2.4)^{*}$ & $3.8(8.3)$ & $4.1(2.1)$ & $2.9(2.5)$ & $0.8(5.9)^{*}$ & $2.3(5.8)$ \\
\hline Range & $1.8-70$ & $1.8-70$ & $3.2-52$ & $2.4-34$ & ND-19 & $1.1-19$ & $0.8-18$ & ND-13 & ND-16 & ND-70 \\
\hline Q1 & 5.9 & 4.3 & 6.9 & 4.8 & 1.8 & - & 3.4 & 1.4 & 0.7 & 1.0 \\
\hline
\end{tabular}


Table 2 continued

\begin{tabular}{|c|c|c|c|c|c|c|c|c|c|c|}
\hline \multirow{2}{*}{$\begin{array}{l}\text { Survey year } \\
\text { No. } \\
\text { Age group (years) }\end{array}$} & \multicolumn{4}{|l|}{1980} & \multicolumn{4}{|l|}{1995} & \multirow{2}{*}{$\begin{array}{l}2003 \\
80\end{array}$} & \multirow{2}{*}{$\begin{array}{l}\text { Total } \\
160\end{array}$} \\
\hline & 40 & $\begin{array}{l}(17) \\
(<40)\end{array}$ & $\begin{array}{l}(13) \\
(40-50)\end{array}$ & $\begin{array}{l}(10) \\
(>50)\end{array}$ & 40 & $\begin{array}{l}(2)^{\mathrm{a}} \\
(<40)\end{array}$ & $\begin{array}{l}(17) \\
(40-50)\end{array}$ & $\begin{array}{l}(21) \\
(>50)\end{array}$ & & \\
\hline Q2 & 10.1 & 10.0 & 11 & 9.1 & 4.2 & - & 4.6 & 2.4 & 1.1 & 2.5 \\
\hline Q3 & 17 & 16 & 24 & 13 & 7.8 & - & 6.8 & 7.9 & 2.1 & 7.7 \\
\hline \multicolumn{11}{|c|}{ CB-163 \& 164 (ng/g lipid) } \\
\hline GM (GSD) & $31(2.7)^{*}$ & $29(2.8)$ & $42(2.5)$ & $24(2.8)$ & $10.3(3.4)^{*}$ & $9.1(11.0)$ & $12(2.9)$ & $9.0(3.7)$ & $3.2(4.1)^{*}$ & $7.5(4.9)$ \\
\hline Range & $4.7-222$ & $7.8-195$ & $12-222$ & $4.7-102$ & ND-117 & $1.7-51$ & $1.2-117$ & ND-86 & $0.1-74$ & ND-222 \\
\hline Q1 & 14 & 13 & 20 & 9.1 & 3.9 & - & 7.8 & 3.2 & 1.2 & 2.0 \\
\hline Q2 & 31 & 29 & 34 & 28 & 12 & - & 13 & 8.8 & 2.7 & 7.8 \\
\hline Q3 & 61 & 57 & 80 & 49 & 27 & - & 25 & 27 & 7.0 & 28 \\
\hline \multicolumn{11}{|l|}{ CB-170 (ng/g lipid) } \\
\hline GM (GSD) & $17(2.4) *$ & $16(2.6)$ & $21(2.4)$ & $15(2.2)$ & $6.4(2.6)^{*}$ & - & $7.2(2.4)$ & $6.1(2.8)$ & $1.6(5.1)^{*}$ & $4.1(5.3)$ \\
\hline Range & $4.3-148$ & $4.4-148$ & $5.1-89$ & $4.3-50$ & ND-53 & ND-24 & $1.4-53$ & ND-33 & ND-25 & ND-148 \\
\hline Q1 & 9.0 & 7.7 & 12 & 8.3 & 2.8 & - & 4.5 & 2.3 & 1.1 & 1.4 \\
\hline Q2 & 18 & 18 & 21 & 18 & 6.1 & - & 6.4 & 4.6 & 1.7 & 4.7 \\
\hline Q3 & 30 & 26 & 37 & 26 & 12 & - & 10.8 & 14 & 3.8 & 13 \\
\hline \multicolumn{11}{|l|}{ CB-180 (ng/g lipid) } \\
\hline GM (GSD) & $60(2.4)^{*}$ & $57(2.6)$ & $73(2.3)$ & $52(2.3)$ & $16(3.1)^{*}$ & $15(9.1)$ & $21(2.5)$ & $13(3.5)$ & $5.8(3.1)^{*}$ & $13(4.2)$ \\
\hline Range & $14-554$ & $16-554$ & $22-306$ & $14-182$ & $1.0-175$ & $3.2-72$ & $3.3-175$ & $1.0-90$ & $0.7-89$ & $0.7-554$ \\
\hline Q1 & 32 & 30 & 38 & 28 & 7.8 & - & 14 & 5.4 & 2.7 & 3.9 \\
\hline Q2 & 60 & 57 & 75 & 66 & 18 & - & 22 & 13 & 4.9 & 14 \\
\hline Q3 & 114 & 91 & 130 & 88 & 37 & - & 33 & 44 & 11 & 43 \\
\hline \multicolumn{11}{|c|}{ CB-182 \& 187 (ng/g lipid) } \\
\hline GM (GSD) & $42(2.7)^{*}$ & $39(2.9)$ & $53(2.4)$ & $34(2.6)$ & $13(3.3)^{*}$ & $11(9.4)$ & $15(3.1)$ & $12(3.4)$ & $5.1(4.0)^{*}$ & $11(4.5)$ \\
\hline Range & $5.7-369$ & $10.9-369$ & $14-268$ & $5.7-130$ & ND-165 & $2.2-52$ & $1.4-165$ & ND-122 & $0.1-105$ & ND-369 \\
\hline Q1 & 20 & 16 & 25 & 21 & 6.1 & - & 10 & 4.1 & 2.2 & 3.3 \\
\hline Q2 & 43 & 32 & 52 & 42 & 13 & - & 16 & 11 & 4.1 & 12 \\
\hline Q3 & 83 & 73 & 100 & 61 & 31 & - & 28 & 39 & 11 & 38 \\
\hline \multicolumn{11}{|c|}{ Penta CBs (ng/g lipid) } \\
\hline GM (GSD) & $122(2.6)^{*}$ & $102(2.7)$ & $179(2.3)$ & $99(2.5)$ & $41(3.1)^{*}$ & $31(17.3)$ & $54(2.1)$ & $34(3.5)$ & $17(3.2)^{*}$ & $35(3.9)$ \\
\hline Range & $18-853$ & $18-559$ & $64-853$ & $24-427$ & $1.8-232$ & $4.1-232$ & $10.4-215$ & $1.8-188$ & $0.6-313$ & $0.6-853$ \\
\hline Q1 & 70 & 44 & 82 & 45 & 20 & - & 36 & 10.5 & 8.7 & 12 \\
\hline Q2 & 134 & 130 & 181 & 113 & 53 & - & 54 & 48 & 15 & 33 \\
\hline Q3 & 228 & 195 & 278 & 185 & 85 & - & 84 & 84 & 33 & 105 \\
\hline \multicolumn{11}{|c|}{ Hexa CBs (ng/g lipid) } \\
\hline GM (GSD) & $275(2.5)^{*}$ & $246(2.8)$ & $371(2.3)$ & $224(2.4)$ & $88(3.5)^{*}$ & $78(13.1)$ & $116(2.6)$ & $72(4.0)$ & $32(3.3)^{*}$ & $71(4.3)$ \\
\hline Range & $55-1788$ & $56-1788$ & $116-1691$ & $55-917$ & $3.0-939$ & $13-483$ & $15-939$ & $3.0-624$ & $2.7-590$ & $2.7-1788$ \\
\hline Q1 & 146 & 116 & 182 & 123 & 41 & - & 83 & 32 & 14 & 21 \\
\hline Q2 & 266 & 265 & 316 & 242 & 103 & - & 119 & 85 & 26 & 73 \\
\hline Q3 & 502 & 469 & 670 & 390 & 237 & - & 215 & 231 & 69 & 238 \\
\hline \multicolumn{11}{|c|}{ Hepta CBs (ng/g lipid) } \\
\hline GM (GSD) & $120(2.5)^{*}$ & $113(2.7)$ & $147(2.3)$ & $102(2.4)$ & $33(3.4)^{*}$ & $28(10.4)$ & $43(2.6)$ & $28(3.9)$ & $13(3.3)^{*}$ & $29(4.3)$ \\
\hline Range & $25-1065$ & $32-1065$ & $41-662$ & $25-362$ & $1.0-393$ & $5.4-148$ & $6.3-393$ & $1.0-237$ & $0.8-209$ & $0.8-1065$ \\
\hline Q1 & 60 & 55 & 72 & 67 & 17 & - & 30 & 12 & 6.3 & 8.3 \\
\hline Q2 & 112 & 107 & 148 & 120 & 36 & - & 43 & 26 & 10.5 & 31 \\
\hline
\end{tabular}


Table 2 continued

\begin{tabular}{|c|c|c|c|c|c|c|c|c|c|c|}
\hline \multirow{2}{*}{$\begin{array}{l}\text { Survey year } \\
\text { No. } \\
\text { Age group (years) }\end{array}$} & \multicolumn{4}{|c|}{1980} & \multicolumn{4}{|c|}{1995} & \multirow{2}{*}{$\begin{array}{l}2003 \\
80\end{array}$} & \multirow{2}{*}{$\begin{array}{l}\text { Total } \\
160\end{array}$} \\
\hline & 40 & $\begin{array}{l}(17) \\
(<40)\end{array}$ & $\begin{array}{l}(13) \\
(40-50)\end{array}$ & $\begin{array}{l}(10) \\
(>50)\end{array}$ & 40 & $\begin{array}{l}(2)^{\mathrm{a}} \\
(<40)\end{array}$ & $\begin{array}{l}(17) \\
(40-50)\end{array}$ & $\begin{array}{l}(21) \\
(>50)\end{array}$ & & \\
\hline Q3 & 221 & 187 & 266 & 175 & 76 & - & 71 & 97 & 27 & 93 \\
\hline
\end{tabular}

In 1980 and 1995, diets were collected in Hokkaido, Tohoku, Kanto, Chubu, Chugoku, Shikoku, Kyushu, and Okinawa

In 2003, diets were collected in Tohoku, Kanto, Chubu, Kansai, Shikoku, and Okinawa

a This group was excluded from statistical comparison due to small number participants

$G M$ geometric mean, GSD geometric standard deviation, $Q 1$ 25th percentile, $Q 2$ median, $Q 3$ 75th percentile

* In all congeners and total PCBs, there are significant differences in log-transformed daily intakes between three survey years $[p<0.05$ by

Tukey's honestly significant difference (HSD) test]. There were no significant differences among the three age groups within the same survey year $(p>0.05)$

(ng/g lipid) according to the age group (Table 3). Furthermore, individual congener groups showed similar increasing trends.

Among the same age groups in the three eras, the serum total PCB level in the younger generation ( $<40$ years) was lower in the 2003 survey than in the 1980 survey. In the age groups of $<40$ years and $40-50$ years, penta CBs were significantly lower in the 2003 survey than in the 1980 survey. The hexa and hepta CBs groups were significantly lower in the $<40$ year age group, but significantly higher in the $>50$ year age group, in the 2003 survey compared with the 1980 survey. These results indicate that hexa and hepta CBs may have accumulated in the $>50$ year age group, whereas all the congeners decreased in the $<40$ year age group, in the 2003 survey compared with the 1980 survey.

Long-term changes in the total PCB levels and congener profiles in participants born from 1940 to 1953

The above results suggested that the age-dependent increases in serum PCB concentrations may be attributable to the accumulation of more highly chlorinated PCBs than lower-chlorinated PCBs. However, testing this hypothesis is not easy because the participants born before 1960 have had more extensive exposure to PCBs than participants born after 1960. Therefore, we compared the serum total PCB levels and congener profiles among the generations born from 1940 to 1953 . Since these birth-year generations were part of all three surveys, the exposure histories can be evaluated. Specifically, females born from 1940 to 1953 ( $n=17$ ) were $<40$ years of age in the 1980 survey, 42-55 years of age in the 1995 survey $(n=17)$, and $50-63$ years of age in the 2003 survey $(n=9)$. The proportions of these serum donors of this generations living in different districts over the three surveys were: TohokuHokkaido, about 20\%; Kanto-Hokuriku-Chubu, about
40\%; Chugoku-Shikoku, about 20\%; and KyushuOkinawa, about $20 \%$.

The serum concentrations and congener profiles are shown in Fig. 1. The serum total PCB levels were significantly increased in 2003. This increase was attributable to a significant increase in the hepta $\mathrm{CB}$ s group. This increase in the hepta CBs group was in contrast with the decrease in the penta CBs group over the 23-year period. These data indicate that the effects of intensive exposure from 1950 to 1970 may have disappeared by 1995 , and therefore may not contribute the large PCB body burden observed in these generations in 2003.

\section{Discussion}

We found age-associated increases in serum PCB levels in females born between 1940 and 1953 in retrospective longterm trend study, although daily intakes have been consistently decreasing. This paradoxical phenomenon has never been reported.

With the advantages of using our samples stored in the specimen bank, the higher serum PCB levels observed in the older generations were found to be correlated with elevation of hepta CBs.

The changes in the congener distribution patterns in serum were in sharp contrast with those in diet. In diet, the congener patterns were almost constant at 1:2:1 (penta:hexa:hepta), despite the fact that total PCB intake decreased significantly from 523 to 63 (ng/day). Since our diet samples covered various kinds of food items, these congener patterns represent the patterns in the modern Japanese diet. Therefore, the age-specific accumulation of hepta CBs in serum is unlikely to be explained by differences in food preferences among generations.

There are at least two possibilities that may explain the age-specific accumulation of hepta CBs in the older 
Table 3 Time era- and age-specific total PCB and congener concentrations in serum

\begin{tabular}{|c|c|c|c|c|c|c|c|c|c|c|}
\hline \multirow{3}{*}{$\begin{array}{l}\text { Survey year } \\
\text { Age (years) } \\
\text { Birth year } \\
\text { (group) } \\
\text { No. }\end{array}$} & \multicolumn{3}{|l|}{1980} & \multicolumn{3}{|l|}{1995} & \multicolumn{3}{|l|}{2003} & \multirow[t]{2}{*}{ Total } \\
\hline & $\begin{array}{l}<40 \\
1946 \\
\text { (group C) }\end{array}$ & $\begin{array}{l}40-50 \\
1936 \\
\text { (group B) }\end{array}$ & $\begin{array}{l}>50 \\
1924 \\
\text { (group A) }\end{array}$ & $\begin{array}{l}<40 \\
1957 \\
\text { (group D) }\end{array}$ & $\begin{array}{l}40-50 \\
1950 \\
\text { (group C) }\end{array}$ & $\begin{array}{l}>50 \\
1934 \\
(\text { group B) }\end{array}$ & $\begin{array}{l}<40 \\
1972 \\
\text { (group E) }\end{array}$ & $\begin{array}{l}40-50 \\
1958 \\
\text { (group D) }\end{array}$ & $\begin{array}{l}>50 \\
1948 \\
\text { (group C) }\end{array}$ & \\
\hline & 17 & 12 & 10 & $2^{\mathrm{a}}$ & 17 & 21 & 59 & 22 & 9 & 169 \\
\hline \multicolumn{11}{|c|}{ Total PCBs (ng/g lipid) } \\
\hline GM (GSD) & $185(1.8)$ & $170(1.4)$ & $125(1.7)$ & $83(2.3)$ & $128(2.0)$ & $164(1.9)$ & $68(1.8) \mathrm{A}$ & $110(1.6) \mathrm{B}$ & $242(1.7) C^{*}$ & $113(2.0)$ \\
\hline Range & $60-414$ & $97-306$ & $55-282$ & $46-148$ & $42-714$ & $67-895$ & $18-282$ & 49-221 & $84-570$ & $18-895$ \\
\hline Q1 & 105 & 118 & 76 & - & 72 & 95 & 43 & 68 & 191 & 66 \\
\hline Q2 & 195 & 180 & 128 & - & 115 & 163 & 65 & 112 & 236 & 114 \\
\hline Q3 & 311 & 220 & 175 & - & 181 & 222 & 106 & 157 & 356 & 182 \\
\hline$\#$ & a & ns & $\mathrm{ns}$ & - & ns & ns & $\mathrm{b}$ & ns & ns & \\
\hline \multicolumn{11}{|c|}{ CB-74 (ng/g lipid) } \\
\hline GM (GSD) & $18(1.9)$ & $17(2.0)$ & $12(1.9)$ & $5.4(2.0)$ & $7.4(1.9)$ & $11(2.1)$ & $3.0(1.9) \mathrm{A}$ & $4.8(1.7) \mathrm{B}$ & $11(1.8) C^{*}$ & $6.5(2.5)$ \\
\hline Range & $6.0-40$ & $6.2-40$ & $7.3-67$ & $3.4-8.8$ & $2.6-33$ & $3.0-51$ & $0.8-12$ & $2.0-12$ & $3.8-29$ & $0.8-67$ \\
\hline Q1 & 10.7 & 8.8 & 8.1 & - & 4.4 & 6.8 & 1.9 & 2.8 & 8.1 & 3.3 \\
\hline Q2 & 17 & 15 & 10.5 & - & 7.1 & 11 & 3.0 & 5.3 & 9.4 & 6.6 \\
\hline Q3 & 33 & 35 & 12 & - & 12 & 18 & 5.0 & 7.6 & 19 & 12 \\
\hline$\#$ & a & a & ns & - & $\mathrm{b}$ & ns & $\mathrm{b}$ & $\mathrm{b}$ & ns & \\
\hline \multicolumn{11}{|c|}{ CB-99 (ng/g lipid) } \\
\hline GM (GSD) & $10.0(1.9)$ & $9.2(1.5)$ & $5.9(1.6)$ & $4.1(1.8)$ & $5.0(2.2)$ & $6.0(2.0)$ & $2.8(1.8) \mathrm{A}$ & $3.9(1.8) \mathrm{A}$ & $7.4(2.1) \mathrm{B}^{*}$ & $4.7(2.2)$ \\
\hline Range & $2.9-26$ & $4.7-16$ & $3.6-18$ & $2.7-6.1$ & $1.1-35$ & $1.2-29$ & $0.9-13$ & $0.9-9.8$ & $1.8-22$ & $0.90-35$ \\
\hline Q1 & 5.6 & 6.3 & 4.6 & - & 3.0 & 3.7 & 1.7 & 2.7 & 4.4 & 2.7 \\
\hline Q2 & 10.6 & 9.2 & 4.9 & - & 4.1 & 6.2 & 2.5 & 4.4 & 7.9 & 4.7 \\
\hline Q3 & 18 & 13 & 7.5 & - & 8.6 & 8.7 & 4.4 & 5.7 & 13 & 7.9 \\
\hline$\#$ & a & $\mathrm{a}$ & ns & - & $\mathrm{b}$ & ns & $\mathrm{b}$ & $\mathrm{b}$ & ns & \\
\hline \multicolumn{11}{|c|}{ CB-118 (ng/g lipid) } \\
\hline GM (GSD) & $26(2.0)$ & $22(1.3)$ & $17(1.8)$ & $8.8(1.7)$ & $12(2.1)$ & $14(2.1)$ & $5.8(1.8) \mathrm{A}$ & 8.9 (1.6)B & $17(2.1) \mathrm{C}^{*}$ & $10.7(2.2)$ \\
\hline Range & $8.4-64$ & $15-37$ & $8.6-53$ & $6.0-13$ & $3.7-107$ & $3.5-79$ & $1.8-16$ & $3.3-17$ & $4.8-56$ & $1.8-107$ \\
\hline Q1 & 13 & 16 & 10.8 & - & 8.2 & 10.2 & 3.7 & 5.8 & 10.1 & 5.8 \\
\hline Q2 & 28 & 23 & 15 & - & 10.9 & 14 & 5.6 & 10.2 & 18 & 10.9 \\
\hline Q3 & 52 & 28 & 23 & - & 17 & 20 & 9.1 & 13 & 31 & 16 \\
\hline$\#$ & a & $\mathrm{a}$ & ns & - & $\mathrm{b}$ & ns & $\mathrm{b}$ & $\mathrm{b}$ & ns & \\
\hline \multicolumn{11}{|c|}{ CB-138 (ng/g lipid) } \\
\hline GM (GSD) & $24(1.9)$ & $23(1.4)$ & $15(1.6)$ & $11(2.1)$ & $15(2.1)$ & $19(2.0)$ & $8.3(1.8) \mathrm{A}$ & $13(1.7) \mathrm{B}$ & $27(1.8) C^{*}$ & $14(2.1)$ \\
\hline Range & $7.8-84$ & $13-45$ & $7.5-33$ & $6.3-18$ & $4.6-121$ & $7.2-167$ & $2.3-31$ & $4.0-27$ & $8.9-64$ & $2.3-167$ \\
\hline Q1 & 13 & 16 & 11 & - & 9.2 & 12 & 5.4 & 8.1 & 20 & 7.9 \\
\hline Q2 & 24 & 24 & 15 & - & 13 & 18 & 7.8 & 14 & 23 & 14 \\
\hline Q3 & 42 & 29 & 22 & - & 24 & 23 & 12 & 20 & 42 & 22 \\
\hline$\#$ & a & a & $\mathrm{ns}$ & - & $a b$ & ns & $\mathrm{b}$ & $\mathrm{b}$ & $\mathrm{ns}$ & \\
\hline \multicolumn{11}{|c|}{ CB-146 (ng/g lipid) } \\
\hline GM (GSD) & $6.1(1.8)$ & $6.0(1.4)$ & $4.2(1.7)$ & $3.1(2.3)$ & $5.1(2.1)$ & $6.6(2.0)$ & $2.6(2.0) \mathrm{A}$ & $4.3(1.7) \mathrm{B}$ & $9.7(1.8) C^{*}$ & $4.2(2.1)$ \\
\hline Range & $2.2-18$ & $3.3-12$ & $1.8-7.3$ & $1.7-5.6$ & $1.7-32$ & $2.5-45$ & $0.5-13$ & $1.5-11$ & $2.8-22$ & $0.5-45$ \\
\hline Q1 & 3.2 & 4.4 & 2.6 & - & 2.9 & 3.5 & 1.6 & 2.9 & 7.5 & 2.6 \\
\hline Q2 & 6.5 & 5.9 & 4.9 & - & 4.7 & 6.3 & 2.5 & 4.4 & 9.9 & 4.5 \\
\hline Q3 & 9.6 & 8.0 & 7.1 & - & 7.4 & 9.5 & 4.5 & 6.2 & 15 & 7.2 \\
\hline$\#$ & a & ns & a & - & ns & $\mathrm{ab}$ & $\mathrm{b}$ & ns & $\mathrm{b}$ & \\
\hline \multicolumn{11}{|c|}{ CB-153 (ng/g lipid) } \\
\hline GM (GSD) & $41(1.8)$ & $40(1.4)$ & $28(1.6)$ & $21(2.3)$ & $33(2.1)$ & $42(2.0)$ & $19(1.9) \mathrm{A}$ & $30(1.6) \mathrm{B}$ & $65(1.7) C^{*}$ & $29(2.0)$ \\
\hline Range & $15-115$ & $25-81$ & $11-53$ & $12-39$ & $11-188$ & $18-295$ & $4.9-85$ & $12-64$ & $23-152$ & $4.9-295$ \\
\hline
\end{tabular}


Table 3 continued

\begin{tabular}{|c|c|c|c|c|c|c|c|c|c|c|}
\hline \multirow{3}{*}{$\begin{array}{l}\text { Survey year } \\
\text { Age (years) } \\
\text { Birth year } \\
\text { (group) } \\
\text { No. }\end{array}$} & \multicolumn{3}{|l|}{1980} & \multicolumn{3}{|l|}{1995} & \multicolumn{3}{|l|}{2003} & \multirow[t]{2}{*}{ Total } \\
\hline & $\begin{array}{l}<40 \\
1946 \\
\text { (group C) }\end{array}$ & $\begin{array}{l}40-50 \\
1936 \\
\text { (group B) }\end{array}$ & $\begin{array}{l}>50 \\
1924 \\
\text { (group A) }\end{array}$ & $\begin{array}{l}<40 \\
1957 \\
\text { (group D) }\end{array}$ & $\begin{array}{l}40-50 \\
1950 \\
\text { (group C) }\end{array}$ & $\begin{array}{l}>50 \\
1934 \\
\text { (group B) }\end{array}$ & $\begin{array}{l}<40 \\
1972 \\
(\text { group E) }\end{array}$ & $\begin{array}{l}40-50 \\
1958 \\
\text { (group D) }\end{array}$ & $\begin{array}{l}>50 \\
1948 \\
\text { (group C) }\end{array}$ & \\
\hline & 17 & 12 & 10 & $2^{\mathrm{a}}$ & 17 & 21 & 59 & 22 & 9 & 169 \\
\hline Q1 & 24 & 28 & 18 & - & 20 & 24 & 12 & 19 & 50 & 18 \\
\hline Q2 & 40 & 41 & 29 & - & 32 & 39 & 18 & 30 & 65 & 29 \\
\hline Q3 & 65 & 51 & 43 & - & 46 & 57 & 28 & 43 & 95 & 47 \\
\hline \# & $\mathrm{a}$ & ns & $\mathrm{a}$ & - & ns & $\mathrm{ab}$ & $\mathrm{b}$ & ns & $\mathrm{b}$ & \\
\hline \multicolumn{11}{|c|}{ CB-156 (ng/g lipid) } \\
\hline GM (GSD) & $5.4(2.0)$ & $4.7(1.4)$ & $3.4(1.6)$ & $2.7(2.6)$ & $4.3(2.3)$ & $5.2(2.0)$ & $1.9(2.0) \mathrm{A}$ & 3.3 (1.7)B & $8.1(1.8) \mathrm{C}^{*}$ & $3.3(2.2)$ \\
\hline Range & $1.9-18$ & $2.6-08$ & $1.5-6.6$ & $1.4-5.4$ & $1.7-48$ & $1.7-39$ & $0.3-6.8$ & $1.5-7.0$ & $2.7-19$ & $0.3-48$ \\
\hline Q1 & 3.0 & 3.6 & 2.3 & - & 2.2 & 3.4 & 1.1 & 1.8 & 5.8 & 1.9 \\
\hline Q2 & 5.0 & 4.9 & 3.7 & - & 3.8 & 5.0 & 1.9 & 3.6 & 8.4 & 3.6 \\
\hline Q3 & 9.1 & 6.3 & 5.3 & - & 6.5 & 7.5 & 3.1 & 4.6 & 12 & 5.5 \\
\hline \# & $\mathrm{a}$ & ns & $\mathrm{a}$ & - & ns & $a b$ & $b$ & ns & $\mathrm{b}$ & \\
\hline
\end{tabular}

CB-163 \& 164 (ng/g lipid)

$\begin{array}{lllllllllll}\text { GM (GSD) } & 10.3(1.9) & 10.3(1.4) & 7.2(1.6) & 4.9(2.3) & 8.2(2.2) & 10.7(2.0) & 4.1(2.0) \mathrm{A} & 6.9(1.6) \mathrm{B} & 16(1.7) \mathrm{C}^{*} & 6.9(2.1) \\ \text { Range } & 3.4-34 & 6.6-20 & 3.0-13 & 2.7-9.0 & 2.9-60 & 4.1-92 & 0.8-17 & 3.0-15 & 5.2-31 & 0.8-92 \\ \text { Q1 } & 5.3 & 7.2 & 4.4 & - & 4.5 & 6.9 & 2.5 & 4.6 & 13 & 4.1 \\ \text { Q2 } & 10.2 & 10.8 & 7.8 & - & 7.8 & 9.2 & 4.1 & 7.0 & 16 & 7.4 \\ \text { Q3 } & 18 & 14 & 11 & - & 12 & 17 & 7.7 & 9.4 & 25 & 11 \\ \# & \mathrm{a} & \mathrm{ns} & \mathrm{a} & - & \mathrm{ns} & \mathrm{ab} & \mathrm{b} & \mathrm{ns} & \mathrm{b} & \end{array}$

CB-170 (ng/g lipid)

$\begin{array}{lllllllllll}\text { GM (GSD) } & 5.8(1.7) & 5.0(1.4) & 4.1(1.7) & 3.4(2.8) & 5.2(2.0) & 6.3(1.8) & 2.8(1.9) \mathrm{A} & 5.1(1.5) \mathrm{B} & 11(1.6) \mathrm{C}^{*} & 4.4(2.0) \\ \text { Range } & 1.9-11 & 2.8-10 & 1.5-6.9 & 1.6-6.9 & 2.2-22 & 2.2-23 & 0.5-12 & 2.6-10 & 4.3-22 & 0.5-23 \\ \text { Q1 } & 3.5 & 3.5 & 2.5 & - & 2.7 & 3.9 & 1.9 & 3.3 & 9.4 & 2.7 \\ \text { Q2 } & 6.0 & 5.3 & 5.1 & - & 4.8 & 6.1 & 2.9 & 5.5 & 10.6 & 4.9 \\ \text { Q3 } & 9.4 & 6.0 & 5.9 & - & 8.2 & 10.2 & 5.0 & 6.7 & 16 & 6.8 \\ \# & \mathrm{a} & \mathrm{ns} & \mathrm{a} & - & \mathrm{ns} & \mathrm{a} & \mathrm{b} & \mathrm{ns} & \mathrm{b} & \end{array}$

CB-180 (ng/g lipid)

$\begin{array}{lllllllllll}\text { GM (GSD) } & 21(1.7) & 19(1.5) & 16(1.8) & 12(2.9) & 19(1.9) & 24(1.8) & 11(1.9) \mathrm{A} & 20(1.5) \mathrm{B} & 46(1.6) \mathrm{C}^{*} & 17(2.0) \\ \text { Range } & 7.3-42 & 10.3-39 & 5.7-28 & 5.9-26 & 7.6-74 & 8.7-69 & 2.8-49 & 10.4-44 & 20-101 & 2.8-101 \\ \text { Q1 } & 14 & 13 & 8.6 & - & 10.2 & 16 & 7.2 & 15 & 35 & 10.6 \\ \text { Q2 } & 24 & 19 & 20 & - & 17 & 23 & 10.7 & 20 & 46 & 19 \\ \text { Q3 } & 31 & 25 & 25 & - & 31 & 42 & 20 & 28 & 59 & 26 \\ \# & \mathrm{a} & \mathrm{ns} & \mathrm{a} & - & \mathrm{ns} & \mathrm{a} & \mathrm{b} & \mathrm{ns} & \mathrm{b} & \end{array}$

CB-182 \& 187 (ng/g lipid)

\begin{tabular}{lllllllllll} 
GM (GSD) & $10.0(1.7)$ & $9.6(1.5)$ & $7.2(1.7)$ & $5.1(2.7)$ & $8.6(1.9)$ & $11(1.9)$ & $5.3(2.0) \mathrm{A}$ & $9.3(1.7) \mathrm{B}$ & $21(1.9) \mathrm{C}^{*}$ & $8.0(2.0)$ \\
Range & $3.7-20$ & $5.4-23$ & $2.8-12$ & $2.5-10.3$ & $2.7-39$ & $4.9-42$ & $0.9-32$ & $3.1-27$ & $6.0-58$ & $0.9-58$ \\
Q1 & 6.5 & 6.5 & 4.1 & - & 5.1 & 6.4 & 3.5 & 7.2 & 15 & 4.9 \\
Q2 & 10.2 & 9.6 & 9.2 & - & 8.7 & 11 & 5.2 & 8.9 & 21 & 8.7 \\
Q3 & 16 & 12 & 11 & - & 14 & 18 & 9.7 & 13 & 31 & 13 \\
$\#$ & $\mathrm{a}$ & $\mathrm{ns}$ & $\mathrm{a}$ & - & $\mathrm{ns}$ & $\mathrm{a}$ & $\mathrm{b}$ & $\mathrm{ns}$ & $\mathrm{b}$ & \\
Penta CBs (ng/g lipid) & & & & & & & & & & \\
GM (GSD) & $55(1.9)$ & $50(1.5)$ & $27(1.8)$ & $18(1.8)$ & $25(2.1)$ & $32(2.0)$ & $12(1.8) \mathrm{A}$ & $18(1.6) \mathrm{B}$ & $36(2.0) \mathrm{C}^{*}$ & $22(2.3)$ \\
Range & $17-122$ & $26-86$ & $10-47$ & $12-28$ & $7.4-175$ & $8.8-126$ & $3.6-36$ & $6.3-35$ & $10.4-108$ & $3.6-175$ \\
Q1 & 29 & 33 & 15 & - & 16 & 22 & 7.2 & 10.9 & 23 & 11 \\
Q2 & 68 & 47 & 34 & - & 23 & 35 & 11 & 19 & 38 & 23 \\
Q3 & 101 & 76 & 42 & - & 39 & 46 & 18 & 26 & 63 & 36 \\
\hline
\end{tabular}


Table 3 continued

\begin{tabular}{|c|c|c|c|c|c|c|c|c|c|c|}
\hline \multirow{3}{*}{$\begin{array}{l}\text { Survey year } \\
\text { Age (years) } \\
\text { Birth year } \\
\text { (group) } \\
\text { No. }\end{array}$} & \multicolumn{3}{|l|}{1980} & \multicolumn{3}{|l|}{1995} & \multicolumn{3}{|l|}{2003} & \multirow[t]{2}{*}{ Total } \\
\hline & $\begin{array}{l}<40 \\
1946 \\
\text { (group C) }\end{array}$ & $\begin{array}{l}40-50 \\
1936 \\
\text { (group B) }\end{array}$ & $\begin{array}{l}>50 \\
1924 \\
\text { (group A) }\end{array}$ & $\begin{array}{l}<40 \\
1957 \\
\text { (group D) }\end{array}$ & $\begin{array}{l}40-50 \\
1950 \\
\text { (group C) }\end{array}$ & $\begin{array}{l}>50 \\
1934 \\
\text { (group B) }\end{array}$ & $\begin{array}{l}<40 \\
1972 \\
\text { (group E) }\end{array}$ & $\begin{array}{l}40-50 \\
1958 \\
\text { (group D) }\end{array}$ & $\begin{array}{l}>50 \\
1948 \\
\text { (group C) }\end{array}$ & \\
\hline & & & 10 & & 17 & 21 & 59 & 22 & & 169 \\
\hline$\#$ & a & $\mathrm{a}$ & $\mathrm{ns}$ & - & $\mathrm{b}$ & $\mathrm{ns}$ & $\mathrm{b}$ & $\mathrm{b}$ & ns & \\
\hline \multicolumn{11}{|c|}{ Hexa CBs (ng/g lipid) } \\
\hline GM (GSD) & $87(1.9)$ & $84(1.4)$ & $58(1.6)$ & $43(2.3)$ & $66(2.1)$ & $84(2.0)$ & $36(1.9) \mathrm{A}$ & $57(1.6) \mathrm{B}$ & $127(1.7) C^{*}$ & $58(2.0)$ \\
\hline Range & $30-265$ & $52-164$ & $25-113$ & $24-77$ & $22-448$ & $35-636$ & $8.9-153$ & $22-119$ & $43-282$ & $8.9-636$ \\
\hline Q1 & 48 & 58 & 38 & - & 39 & 48 & 23 & 34 & 99 & 35 \\
\hline Q2 & 87 & 89 & 59 & - & 61 & 79 & 33 & 56 & 129 & 56 \\
\hline Q3 & 145 & 106 & 89 & - & 93 & 109 & 54 & 83 & 192 & 92 \\
\hline$\#$ & a & ns & $\mathrm{a}$ & - & ns & $a b$ & $\mathrm{~b}$ & ns & $\mathrm{b}$ & \\
\hline \multicolumn{11}{|c|}{ Hepta CBs (ng/g lipid) } \\
\hline GM (GSD) & $37(1.7)$ & $34(1.5)$ & $35(1.8)$ & $21(2.8)$ & $33(1.9)$ & $42(1.8)$ & 19 (1.9)A & $35(1.6) \mathrm{B}$ & $78(1.6) C^{*}$ & $30(2.0)$ \\
\hline Range & $13-70$ & $19-71$ & $20-138$ & $10-43$ & $13-131$ & $16-134$ & $4.2-94$ & $18-76$ & $31-181$ & $4.2-181$ \\
\hline Q1 & 24 & 23 & 23 & - & 18 & 27 & 13 & 24 & 63 & 18 \\
\hline Q2 & 39 & 35 & 30 & - & 30 & 39 & 19 & 35 & 75 & 34 \\
\hline Q3 & 58 & 44 & 42 & - & 53 & 70 & 35 & 45 & 106 & 45 \\
\hline \# & $\mathrm{a}$ & ns & $\mathrm{a}$ & - & ns & $\mathrm{a}$ & $\mathrm{b}$ & ns & $\mathrm{b}$ & \\
\hline
\end{tabular}

$G M$ geometric mean, GSD geometric standard deviation, $Q 1$ 25th percentile, $Q 2$ median, $Q 3$ 75th percentile

a This group was excluded from statistical comparison due to small number participants

* There were significant differences among the three age groups within the same survey year $(p<0.05$ by Tukey's HSD test). Values with a different capital letter differ significantly from each other. For example, a value indicated by A differs from the corresponding values indicated by $\mathrm{B}$ or $\mathrm{C}$

\# Comparison among the three surveys within the same age group. Values with a different lower-case letter differ significantly from each other

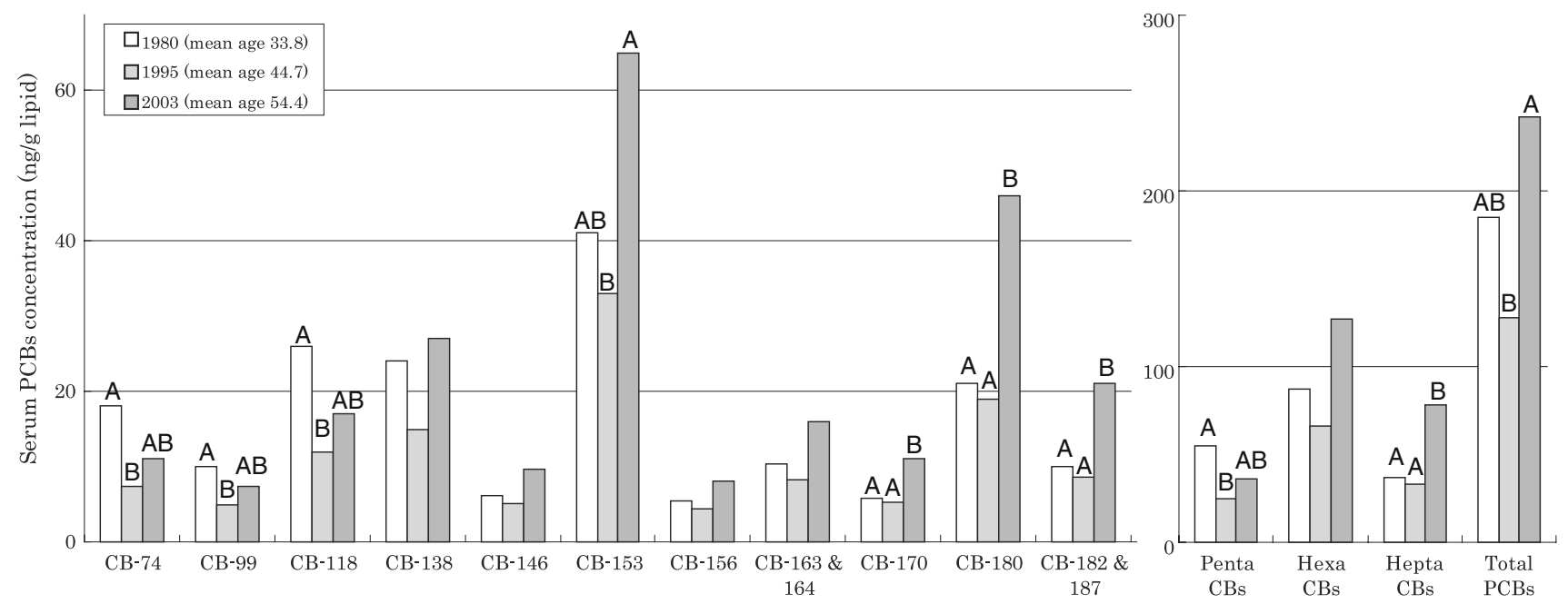

Fig. 1 Serum concentrations of PCBs (ng/g lipid) in females born from 1940 to 1953 in the three different surveys (1980, 1995, and 2003). The letters indicate the results of statistical analyzes by ANOVA. When the ANOVA was significant, Tukey's post hoc tests

generations. The first hypothesis assumes a birth-year generation phenomenon, involving generation-specific exposure histories to PCBs. Since highly chlorinated PCBs are more lipophilic and have longer half-lives [22], the were conducted. The letters $A$ and $B$ indicate that the corresponding values differ significantly at $p<0.05$, while $A$ and $A B$ or $A B$ and $B$ do not

apparent accumulation of highly chlorinated PCBs in the older generation may be associated with past intense exposure from 1950 to 1970. Alternatively, the second hypothesis assumes a decrease in elimination of highly 
chlorinated PCBs with age. It is well established that PCB metabolic activity is mediated by P450s [12-15], which is depressed with aging [16].

The two hypotheses were tested by long-term observations in generations born from 1940 to 1953 in various geographic locations. The results indicated preferential increases in the hexa and hepta concentrations in 2003 compared with the corresponding levels in 1995 and 1980. Although these generations had been extensively exposed to PCBs, an effect of this high exposure history was only discernible in the 1980 survey and had disappeared in the 1995 survey. Thus, the recent preferential increases in hepta CBs despite large decreases in dietary PCB intake suggest that the accumulation of these congener groups is probably due to decreased elimination with aging, rather than a birth-generation phenomenon or preferred consumption of fish.

The present findings may raise a new issue for risk assessment of PCBs. Neonates and children have been postulated to be the most susceptible to exposure to PCBs [23]. However, since it is well known that exposure to PCBs is associated with various cancers [24-29], older generations may need to be considered as a high-risk population due to their tendency for higher accumulation of PCBs than younger generations.

The present study has several limitations. First, it was not a cohort study. Thus, the present observations may be confounded by both individual and geographical differences. The present population was, however, free from occupational exposure. The second limitation is the absence of individual background data concerning number of pregnancies, number of breast feeding, body mass index (BMI), age of menopause, and consumption of fish, which are known to be determinants for serum PCB levels: number of pregnancies [30], breastfeeding [31, 32], BMI [33], age of menopause [30], and consumption of fish [34]. The third limitation is uncertainty about the sources of the PCBs. We assumed that the major source of PCBs is diet. However, inhalation is well known to be another important exposure route [35], and we did not evaluate exposure through this route. Our neglect of routes other than food may be justifiable because more than $90 \%$ of exposure occurs via food [36].

In conclusion, the advantages of using historical samples superseded the various limitations of this study. The versatile evidence, although indirect, strongly suggests preferential accumulation of highly chlorinated PCBs in the older generation. Such generation-specific accumulation warrants further investigation.

Acknowledgments This study was primarily supported by the Japan Science and Technology Agency (No. 1300001) and a grant-in- aid for Health Science Research from the Ministry of Health, Labor, and Welfare of Japan (H15-Chemistry-004, H-21-Food-General-003).

\section{References}

1. Solomon GM, Weiss PM. Chemical contaminants in breast milk: time trends and regional variability. Environ Health Perspect. 2002;110:A339-47.

2. Sjodin A, Jones RS, Focant JF, Lapeza C, Wang RY, McGahee EE 3rd, et al. Retrospective time-trend study of polybrominated diphenyl ether and polybrominated and polychlorinated biphenyl levels in human serum from the United States. Environ Health Perspect. 2004;112:654-8.

3. Dachs J, Lohmann R, Ockenden WA, Mejanelle L, Eisenreich SJ, Jones KC. Oceanic biogeochemical controls on global dynamics of persistent organic pollutants. Environ Sci Technol. 2002; 36:4229-37.

4. Watanabe T, Zhang ZW, Moon CS, Shimbo S, Nakatsuka H, Matsuda-Inoguchi $\mathrm{N}$, et al. Cadmium exposure of women in general populations in Japan during 1991-1997 compared with 1977-1981. Int Arch Occup Environ Health. 2000;73:26-34.

5. Watanabe T, Koizumi A, Fujita H, Kumai M, Ikeda M. Dietary cadmium intakes of farmers in nonpolluted areas in Japan, and the relation with blood cadmium levels. Environ Res. 1985; 37:33-43.

6. Koizumi A, Yoshinaga T, Harada K, Inoue K, Morikawa A, Muroi J, et al. Assessment of human exposure to polychlorinated biphenyls and polybrominated diphenyl ethers in Japan using archived samples from the early 1980s and mid-1990s. Environ Res. 2005;99:31-9.

7. DeCaprio AP, Johnson GW, Tarbell AM, Carpenter DO, Chiarenzelli JR, Morse GS, et al. Polychlorinated biphenyl (PCB) exposure assessment by multivariate statistical analysis of serum congener profiles in an adult Native American population. Environ Res. 2005;98:284-302.

8. Bates MN, Buckland SJ, Garrett N, Ellis H, Needham LL, Patterson DG Jr, et al. Persistent organochlorines in the serum of the non-occupationally exposed New Zealand population. Chemosphere. 2004;54:1431-43.

9. De Saeger S, Sergeant H, Piette M, Bruneel N, Van de Voorde W, Van Peteghem C. Monitoring of polychlorinated biphenyls in Belgian human adipose tissue samples. Chemosphere. 2005; 58:953-60.

10. Apostoli P, Magoni M, Bergonzi R, Carasi S, Indelicato A, Scarcella C, et al. Assessment of reference values for polychlorinated biphenyl concentration in human blood. Chemosphere. 2005;61:413-21.

11. Masuda Y, Haraguchi K, Kono S. Peculiar remaining of some PCB congeners in the patients with Yusho for more than 30 years. Fukuoka Igaku Zasshi. 2003;94:136-43.

12. Safe S. Toxicology, structure-function relationship, and human and environmental health impacts of polychlorinated biphenyls:progress and problems. Environ Health Perspect. 1992; 100:259-68.

13. Brown JF Jr, Lawton RW, Morgan CB. PCB metabolism, persistence, and health effects after occupational exposure: implications for risk assessment. Chemosphere. 1994;29:2287-94.

14. Ariyoshi N, Oguri K, Koga N, Yoshimura H, Funae Y. Metabolism of highly persisitent PCB congener, 2,4,5,2 $2^{\prime}, 4^{\prime}, 5^{\prime}$-hexachlorobiphenyl, by human CYP2B6. Biochem Biophys Res Commun. 1995;212:455-60.

15. Hovander L, Malmberg M, Athanasiadou I, Rahm S, Bergman A, Klasson Wheler E. Identification of hydroxylated PCB 
metabolites and other phenolic halogenated pollutants in human blood plasma. Arch Environ Contam Toxicol. 2002;42:105-17.

16. Kinirons MT, O'Mahony MS. Drug metabolism and ageing. $\mathrm{Br}$ J Clin Pharmacol. 2004;57:540-4.

17. Gladen BC, Doucet J, Hansen LG. Assessing human polychlorinated biphenyl contamination for epidemiologic studies: lessons from patterns of congener concentrations in Canadians in 1992. Environ Health Perspect. 2003;111:437-43.

18. Hites RA. Polybrominated diphenyl ethers in the environment and in people: a meta-analysis of concentrations. Environ Sci Technol. 2004;38:945-56.

19. Hirai T, Fujimine Y, Watanabe S, Nakano T. Congener-specific analysis of polychlorinated biphenyl in human blood from Japanese. Environ Geochem Health. 2005;27:65-73.

20. Phillips DL, Pirkle JL, Burse VW, Bernert JTJ, Henderson LO, Needham LL. Chlorinated hydrocarbon levels in human serum: effects of fasting and feeding. Arch Environ Contam Toxicol. 1989;18:495-500.

21. Chiba K, Koizumi A, Kumai M, Watanabe T, Ikeda M. Nationwide survey of high-density lipoprotein cholesterol among farmers in Japan. Prev Med. 1983;12:508-22.

22. Shirai JH, Kissel JC. Uncertainty in estimated half-lives of PCBS in humans: impact on exposure assessment. Sci Total Environ. 1996;187:199-210.

23. Winneke G, Walkowiak J, Lilienthal H. PCB-induced neurodevelopmental toxicity in human infants and its potential mediation by endocrine dysfunction. Toxicology. 2002;181-182:161-5.

24. Laden F, Ishibe N, Hankinson SE, Wolff MS, Gertig DM, Hunter DJ, et al. Polychlorinated biphenyls, cytochrome P450 1A1, and breast cancer risk in the Nurses' Health Study. Cancer Epidemiol Biomarkers Prev. 2002;11:1560-5.

25. Mallin K, McCann K, D'Aloisio A, Freels S, Piorkowski J, Dimos J, et al. Cohort mortality study of capacitor manufacturing workers, 1944-2000. J Occup Environ Med. 2004;46:565-76.

26. Verkasalo PK, Kokki E, Pukkala E, Vartiainen T, Kiviranta H, Penttinen A, et al. Cancer risk near a polluted river in Finland. Environ Health Perspect. 2004;112:1026-31.
27. Zhang Y, Wise JP, Holford TR, Xie H, Boyle P, Zahm SH, et al. Serum polychlorinated biphenyls, cytochrome P-450 1A1 polymorphisms, and risk of breast cancer in Connecticut women. Am J Epidemiol. 2004;160:1177-83.

28. Ritchie JM, Vial SL, Fuortes LJ, Robertson LW, Guo H, Reedy VE, et al. Comparison of proposed frameworks for grouping polychlorinated biphenyl congener data applied to a case-control pilot study of prostate cancer. Environ Res. 2005;98:104-13.

29. Moysich KB, Shields PG, Freudenheim JL, Schisterman EF, Vena JE, Kostyniak P, et al. Polychlorinated biphenyls, cytochrome P4501A1 polymorphism, and postmenopausal breast cancer risk. Cancer Epidemiol Biomarkers Prev. 1999;8:41-4.

30. Moysich KB, Ambrosone CB, Mendola P, Kostyniak PJ, Greizerstein HB, Vena JE, et al. Exposures associated with serum organochlorine levels among postmenopausal women from western New York State. Am J Ind Med. 2002;41:102-10.

31. Duarte-Davidson R, Jones KC. Polychlorinated biphenyls (PCBs) in the UK population: estimated intake, exposure and body burden. Sci Total Environ. 1994;151:131-52.

32. Sandanger TM, Brustad M, Odland JO, Doudarev AA, Miretsky GI, Chaschin V, et al. Human plasma levels of POPs, and diet among native people from Uelen, Chukotka. J Environ Monit. 2003;5:689-96.

33. Glynn AW, Granath F, Aune M, Atuma S, Darnerud PO, Bjerselius R, et al. Organochlorines in Swedish women: determinants of serum concentrations. Environ Health Perspect. 2003;111:349-55.

34. Hagmar L, Wallin E, Vessby B, Jonsson BA, Bergman A, Rylander L. Intra-individual variations and time trends 19912001 in human serum levels of PCB, DDE and hexachlorobenzene. Chemosphere. 2006;64:1507-13.

35. Breivik K, Alcock R, Li YF, Bailey RE, Fiedler H, Pacyna JM. Primary sources of selected POPs: regional and global scale emission inventories. Environ Pollut. 2004;128:3-16.

36. Liem AKD, Theelen RMC, Dioxins: chemical analysis, exposure and risk assessment [Doctor thesis Thesis]. University of Utrecht, Utrecht. 